\title{
Médiévales
}

Langues, Textes, Histoire

45 | automne 2003

Grammaires du vulgaire

\section{Les clercs et la langue romane : une boutade renardienne au XIV ${ }^{\mathrm{e}}$ siècle}

Clerics and Romance Languages: a 14th Century Jest from Reinart the Fox

\section{Jean Batany}

\section{OpenEdition}

\section{Journals}

Édition électronique

URL : https://journals.openedition.org/medievales/767

DOI : 10.4000/medievales.767

ISSN : 1777-5892

\section{Éditeur}

Presses universitaires de Vincennes

\section{Édition imprimée}

Date de publication : 16 septembre 2003

Pagination : 85-98

ISBN : 2-84292-146-1

ISSN : 0751-2708

\section{Référence électronique}

Jean Batany, « Les clercs et la langue romane : une boutade renardienne au xive siècle », Médiévales [En ligne], 45 | automne 2003, mis en ligne le 09 décembre 2005, consulté le 24 avril 2022. URL : http:// journals.openedition.org/medievales/767 ; DOI : https://doi.org/10.4000/medievales.767

Ce document a été généré automatiquement le 24 avril 2022

Tous droits réservés 


\title{
Les clercs et la langue romane : une boutade renardienne au XIV ${ }^{\mathrm{e}}$ siècle
}

\author{
Clerics and Romance Languages : a 14th Century Jest from Reinart the Fox
}

Jean Batany

1 Un des points centraux de notre objet est l'accès du français au statut de langue de culture, puisque c'est à ce titre que nous avons, bon gré mal gré, à en enseigner l'histoire, en dépit de notre rêve impossible de linguistes qui voudraient faire accéder à la réalité quotidienne de la communication au temps passé. Une étape importante de cette promotion du français peut se situer au début du XIve siècle, bien que cela ne coïncide pas en France avec des chefs-d'œuvre littéraires indiscutés. Il faut reconnaître qu'à cette époque, la notion de « culture » est principalement évoquée par les mots clerc et clergie, qui se réfèrent, en principe, assez directement à la réalité d'un statut social peut-être, il est vrai, en cours d'évolution.

2 Il n'est donc pas artificiel de monter en épingle un texte très bref, tiré d'une œuvre dont la perspective est plus distrayante que didactique, et qui met une formule plaisante dans la bouche d'un personnage ambigu, pour affecter de définir, avec une autorité comique, un certain rapport entre la langue française et le statut de clerc, statut dont l'auteur prétend justement avoir bénéficié avant d'en être exclu. Prenons cet auteur tel qu'il se présente, tant pis si son curriculum vitae est truqué par lui ou même imaginaire. Ayant fait des études assez poussées dans les écoles, il a dû accéder à un grade du clergé que nous ne savons pas exactement, puis, grâce en partie à sa culture, il a fait fortune à Troyes comme épicier-apothicaire, statut marginal entre les "arts mécaniques" et les sciences "libérales", ce qui le place peut-être à un point d'observation focal pouvant lui permettre des positions ambivalentes ${ }^{1}$. Mais, à la suite de ses activités séculières, il aurait été exclu des privilèges des clercs, dans des conditions sur lesquelles on pourra revenir, tout en conservant ou en acquérant une énorme bibliothèque, latine et française, dont il semble avoir voulu faire profiter des lecteurs moins informés, en la déversant dans un cadre distrayant que nous pouvons considérer comme un parcours d'accès pédagogique à la morale et aux connaissances intellectuelles - à moins d'y voir (nous y reviendrons), une dérision radicale de ce 
monde de la "clergie». L'ouvrage ainsi composé constitue le Roman de Renart le Contrefait, qui comporte deux versions successives, écrites entre 1320 et 1342 (la première version comprenant 32000 vers, la seconde 41150 vers, coupés en outre par une longue digression historique en prose) ${ }^{2}$. Le cadre général est, en principe, celui des aventures de Renart, thème déjà connu à l'époque par les récits dont plusieurs manuscrits antérieurs ou contemporains s'intègrent à ce que nous appelons le Roman de Renart. Des histoires d'animaux distrayantes restent donc la base des différentes "branches" du texte; mais celui-ci, surtout dans le seconde version, y intègre, généralement sous forme de discours des personnages, de très longs développements historiques, moraux ou érudits, dont on peut souvent identifier les sources, latines ou françaises, parfois reproduites quasi-textuellement, comme certains passages du Roman de la Rose ou du Livre de Philosophie et de Moralité d'Alart de Cambrai.

Les récits distrayants de type renardien, dont la place est particulièrement importante dans la première version, sont rarement de simples doublets du Roman de Renart proprement dit. Ils puisent en particulier dans la tradition des fables ésopiques ${ }^{3}$. Un passage de la première version, non repris tel quel dans la seconde, raconte ainsi une histoire qui constitue, sous des variantes diverses, une fable très répandue, puisqu'on la trouvera sous trois formes dans l'Ésope de Julien $\mathrm{Macho}^{4}$. Son schéma typique présente un prédateur, lion ou loup, qui prétend dévorer une proie: un âne, un cheval, une jument, ou plutôt le petit de la jument. Il aborde l'équidé avec son désir cynique, ou en affectant des talents de médecin ; l'animal lui tend son sabot pour qu'il en extraie une épine ou qu'il y lise quelque chose; le prédateur regarde alors de près, et se fait assommer d'un grand coup de sabot dans la gueule ${ }^{5}$. Dans la version de Renart le Contrefait, le loup, Ysengrin, vient dire à la jument qu'il veut manger son poulain nouveau-né ; elle répond qu'elle est d'accord, à condition que l'enfant soit d'abord baptisé. Ysengrin accepte de le baptiser, mais il veut savoir quel nom lui donner; la jument prétend qu'il n'a qu'à lire le nom en dessous de son sabot. La variante originale de notre texte, c'est qu'Ysengrin est en compagnie de Renart, et qu'à ce moment, ne sachant pas bien lire, il demande à son ami, plus savant, de venir l'aider. Renart se récuse avec deux arguments : d'abord, il est très enrhumé et ne voit pas bien clair; et surtout, il n'a fait que des études juridiques (donc en latin) et ne sait pas lire le français ; vous trouverez, dit-il, difficilement (« anviz », du lat. « invitus ») un bon clerc qui sache bien lire ou écrire un texte roman:

Et si n'ai je lehu qu'an lois ;

Si ne sai point lire françois!

Anviz verroiz bon clerc bien lire

En nul romant, ne bien escrire. ${ }^{6}$

4 Ysengrin doit donc se débrouiller tout seul, et reçoit le coup de sabot. Renart va se cacher dans une haie (comme il fait souvent pour jouir de la détresse de ses victimes). Ici, je crois qu'on pourrait mettre dans la bouche de Renart, avec des guillemets, la première "moralité » proposée après l'aventure, et prêtée par les éditeurs au narrateur :

« Miaux li vausist estre an maison!

Adés n'a pas clergié saison:

Or voy ge bien tout en apert

Que clergié bien sa saison pert :

Aucunes foiz vilain gueaignent

Es leus ou li clerc se mehainnent : 
S'Isangrin lire ne saüst,

Ancor ceste prune il n'eüst ».7

Un autre commentaire est explicitement mis ensuite dans la bouche du loup :

«Ge ne fis mie grant savoir

Quant ge vouloie clers devenir ;

Ge m'an sai bien a coi tenir :

Tel mestier n'ai ge mie chier,

Ge ain trop miax estre bouchier ${ }^{8}{ }^{8}$

6 Ces commentaires glissent, de l'un à l'autre, vers le thème déjà banal dans la littérature zoomorphique du loup qui veut apprendre à lire, entrer dans les ordres, ou accomplir un acte liturgique, et qui échoue lamentablement. Ce qui est plus original, c'est l'insistance de Renart sur la notion de clerc, et surtout sa première réplique sur la lecture du français.

7 Cette originalité apparaît en comparant notre histoire à celle de la chèvre Barbue, qui figure dans la "première branche », et que la seconde version de l'ouvrage conserve, alors qu'elle supprime l'histoire de la jument ${ }^{9}$. La chèvre a un conflit juridique avec Ysengrin sur ses droits de pâture, et elle prétend apporter un parchemin prouvant son bon droit ; le loup, comptant gagner le procès et manger la chèvre, persuade Renart de l'aider grâce à sa clergie; en fait, le parchemin ne contient aucun texte lisible, mais Renart a vu les deux chiens que Barbue a recrutés pour la sauver, et il prétend que le document donne raison à la chèvre. Cette fable, comme celle de la jument, est donc fondée sur le principe d'une lecture impossible pour le loup ignorant, et pour laquelle Renart le clerc se défausse ; mais il n'y est pas du tout question de distinguer entre latin et français. En supprimant l'histoire de la jument de la seconde version, notre auteur at-il senti confusément que l'opposition entre latin et langue vulgaire l'avait engagé dans une problématique dangereuse?

8 Il est difficile de déchiffrer les intentions d'un écrivain qui bavarde à tort et à travers. Risquons quelques réflexions provisoires, en juxtaposant des questions que notre texte semble poser: le choix d'une problématique au niveau de l'écrit et non de l'oral; l'inversion des niveaux de difficulté normalement admis (le français serait plus difficile que le latin !) ; la position du problème à propos du clerc, et non de l'ensemble de la société; le contexte situant la question dans l'atmosphère du baptême et de la maternité (ce qui peut ouvrir des perspectives sur les rapports des deux cultures avec la féminité); enfin (et au risque de démolir les réflexions précédentes), l'ambiguïté fondamentale du texte, à prendre au sérieux ou au comique, à la lettre ou au second degré, au sens direct ou par antiphrase...

Une problématique de l'écrit

9 Renart peut se dérober parce que lui, le détenteur par excellence de la parole, on l'appelle au secours pour déchiffrer un écrit. Ayant fait des études, il a dû lire, bien sûr, mais seulement « en lois».

10 L'idéalisation du modèle écrit est une vieille obsession du Moyen Âge, paradoxalement contraignante comme mauvaise conscience aux $\mathrm{x}^{\mathrm{e}}-\mathrm{XI}^{\mathrm{e}}$ siècles, où règne la préférence pour les témoignages oraux. Mais il ne faut pas trop opposer les deux formes : l'écrit est un enregistrement ou un aide-mémoire occasionnel du discours oral, l'oral est senti comme le déchiffrement d'un écrit réel ou plus souvent virtuel ${ }^{10}$. Si l'on relit l'excellent livre de S. Lusignan, Parler vulgairement, on constate qu'en fin de compte, la plupart des textes qu'il invoque se réfèrent aux idiomes vulgaires comme à des parlers, sans 
envisager leur forme écrite, sauf dans les introductions à des traductions, et dans certains manuels d'apprentissage du français ${ }^{11}$. Pourtant, il a bien été établi, par Beaulieux et après lui, que le français s'impose comme langue écrite à cette époque (fin XIII-début XIV ${ }^{\mathrm{e}}$ siècle), non pas tant par la littérature, mais par la pratique juridique et administrative : ce sont les notaires qui ont fait le français, et non pas les écrivains. Dans notre texte, il s'agit bien d'un usage de la pratique: l'espèce de contrat que constitue le baptême, et qui est représenté éminemment par le nom, se voit matérialisé par l'écriture de ce nom : en quelque sorte, le droit s'inscrit sous le sabot de la jument, qui ne se reconnaît pas la capacité de le déclarer oralement (évidence juridique qu'Ysengrin ne discute pas). Nous sommes donc fort loin du De vulgari eloquentia de Dante (texte qui, du reste, n'était pas connu en France avant 1350): certes, en envisageant les diverses formes de langue comme des parlers, Dante montre souvent qu'il s'intéresse à des textes qu'il a connus sous forme écrite, mais il s'agit de textes littéraires, relevant surtout de la poésie lyrique ${ }^{12} ; c^{\prime}$ est encore plus net dans le Convivio : l'emploi écrit des parlers vulgaires pour la pratique juridique et administrative ne l'intéresse pas. En fait, il situe l'écrit par rapport à des idéaux d'ordre vocal: l'éloquence et le chant.

11 En somme, Dante méprise plus ou moins un art d'écrire qui ne serait pas rhétorique ou lyrique : la primauté qu'il reconnaît au français, du bout des lèvres, dans le domaine de la prose, nous fait un peu sourire ${ }^{13}$. Notre Renart ne semble pas du tout avoir ce partipris, mais il ne se montre pas, à l'opposé, un vulgaire "praticien». Sans doute, il n'envisage pas l'art de «dictier», terme qui va désigner longtemps la composition proprement littéraire, mais son expression « ne bien escrire » déborde probablement sur la technique du scribe : il s'agit de l'art de rédiger, dont les aspects matériels ne sont pas bien dissociés de ses aspects culturels. Celui qui écrit ordinairement en latin est-il embarrassé quand il s'agit d'écrire en français?

On pourrait ici reprendre l'étude précise des manuscrits bilingues, non seulement les manuscrits littéraires ${ }^{14}$, mais ceux de la pratique, sans négliger le point de vue paléographique : un même scribe, en passant du latin au français ou inversement, peut avoir des hésitations intéressantes sur les questions d'orthographe, d'abréviation, ou même de ductus des lettres. Il a pu être surtout embarrassé pour écrire ce qu'il entendait en langue vulgaire d'après des modèles hésitants de transcription, par la polysémie des graphèmes et la polygraphie des phonèmes éparpillées au XIV siècle, aussi loin de la normalisation qui avait été tentée au temps de Guiot de Provins que de celle qui tendra à se réaliser au $\mathrm{Xv}^{\mathrm{e}}$ siècle ${ }^{15}$. Notre épicier de Troyes peut donc prêter à son Renart une observation qu'il a pu faire devant la maladresse de certains notaires, parfois ses anciens condisciples, transcrivant des textes réglementaires ou judiciaires qui intéressaient son travail commercial. Mais l'adverbe «bien » qualifiant " escrire » vise évidemment, au-delà du niveau technique, une sorte d'idéal moral ou esthétique, ou du moins le sentiment d'un bien-être dans l'exercice d'une activité.

Une hiérarchie paradoxale des difficultés

Il faut admettre, en effet, que notre ex-clerc se sentait plus à l'aise que ses ex-confrères en écrivant en français, et qu'il considérait avec ironie leur embarras, parce que cet embarras contredisait le principe admis à l'époque dans la tradition scolaire : le latin représente un niveau de difficulté supérieur à celui de la langue vulgaire. Rappelons, par exemple, que les deux premières attestations de l'expression "sermo maternus ", au début du XII ${ }^{\mathrm{e}}$ siècle ${ }^{16}$, servaient à Guibert de Nogent pour s'étonner de la capacité du 
pape Urbain II (dans un cas) et de la sienne propre (dans l'autre) à parler latin aussi aisément que leur langue maternelle française. Dans une situation de diglossie (comme dans toute structure de couple, fût-elle matrimoniale ou d'alliance politique...), la dualité est nécessairement liée à une hiérarchie, où la facilité prétendument "enfantine" d'un des groupes peut définir son infériorité. La rigueur de l'enseignement aux XIV ${ }^{e}-\mathrm{XV}^{\mathrm{e}}$ siècles entraîne (c'est bien attesté au temps de Gerson) la punition des élèves qui parlent français et non latin (avec des techniques pédagogiques analogues à celle du "mistigri » cinq siècles plus tard, quand l'écolier flamand, provençal ou africain devra passer son bâton de punition à celui qu'il surprendra à ne pas parler la « langue de culture » que sera devenu le français).

Dans une telle situation de diglossie, la "méthode directe " a peut-être réussi à Montaigne (encore qu'on doute du résultat, car il n'a pas publié de grande œuvre en latin!), mais elle s'est avérée catastrophique ailleurs, en particulier dans les colonies françaises ${ }^{17}$. Les documents étudiés par S. Lusignan montrent qu'on a heureusement, aux XIII ${ }^{e}$ et XIV ${ }^{e}$ siècles, pris le parti de faire travailler l'écolier à partir de sa langue maternelle pour l'amener progressivement à la grammatica $^{18}$. La langue vulgaire a dû être plus ou moins utilisée au niveau du trivium ; cependant, son emploi semble discuté au-delà de la rhétorique. Roger Bacon affirme la quasi-impossibilité d'exprimer les notions de la logique dans la langue maternelle ${ }^{19}$; mais, dans un texte que S. Lusignan ne cite pas (il est vrai qu'il ne s'agit pas du français), Raymond Lulle semble protester contre cette idée : "Avant d'apprendre la logique en latin, tu l'apprendras en roman, avec les rimes qui sont après ce livre ; et sais-tu pourquoi ? Parce qu'ainsi tu la sauras plus tôt en latin et tu l'entendras mieux $»^{20}$.

Les traducteurs du XIV ${ }^{e}$ siècle reprennent et précisent pourtant les idées de Roger Bacon sur la difficulté d'exprimer certaines notions en langue vulgaire ${ }^{21}$. C'est surtout en ce sens, pour eux, que l'usage du roman est difficile. Mais il ne semble pas qu'ils seraient opposés à faire partir les enfants des règles d'usage de leur langue maternelle pour leur enseigner la grammatica. En fait, le problème de la progression du français au latin pour les écoliers est différent du problème posé à un niveau supérieur pour passer des structures intellectuelles, exprimées en latin, à une forme d'expression saisie dans la communication : sur ce plan, l'enfermement dans le latin risque d'être une source de stagnation intellectuelle; le passage à la langue vulgaire pourrait déterminer un affinement de la communication, et par là, de la connaissance elle-même.

Notre auteur veut-il dire cela? Il ne le semble pas, si l'on regarde ce qu'il fait dire à Renart lui-même dans son cours d'histoire universelle ${ }^{22}$ : quand Énée arrive en Italie, c'est chez le roi Latin, qui « trouva latin premierement [...]/Et tant s'estudie y mist/Que le latin proprement fist ». Ce latin qui, nous dit-on en quarante-deux vers, est la base de toute science et de tous les " arts ", mettant ainsi le roi Latin au niveau de Noé " qui la vigne planta». Quelle que soit la source de ce jeu éponymique, inconnu des commentaires virgiliens ${ }^{23}$, cette idée de le transférer d'un peuple à une langue est une occasion, pour Renart, de discourir sur la supériorité de la « clergie » - bien que ce mot n'y figure pas...

17 En fait, l'éloge du latin s'achève sur une ironie satirique : «La Cour de Rome la scet bien : / Du latin naist ou mal ou bien » : c'est-à-dire que le latin sert, dans un procès à la Curie, à faire « du vrai le faux ou du faux le vrai », comme la logique dans un Mariage des sept $\operatorname{arts}^{24}$. Mais la longue tirade ne peut être construite uniquement pour cette chute. Roger Bacon estimait qu'il fallait chercher en amont du latin, en apprenant le grec, 
l'hébreu et l'arabe, où les sciences s'étaient d'abord développées, le latin, langue banale de la culture, ne faisant guère qu'offrir des traductions ${ }^{25}$. On pourrait penser, à la rigueur, que pour Renart, le français, langue trop banale, doit être dépassé en amont par un recours au latin, qui jouerait le rôle du grec ou de l'arabe pour Roger Bacon, mais cela ne ressort guère du texte. La confrontation de l'épisode de Latinus et de celui de la jument semble donc orienter plutôt vers l'idée que toute « clergie » comporte un risque lié à sa valeur même et à ses difficultés, la supériorité profonde du latin ne l'en protégeant pas. Le problème du statut du clerc semble donc plus profond que celui du statut de chaque langue.

Le clerc, l'écriture et les langues

18 À la différence de Dante, notre auteur situe nettement son problème au niveau du " clerc ». Mais cette notion est en pleine crise à l'époque. On est clerc quand on a fait des études, en principe dans un milieu ecclésiastique et en latin; la cléricature est un rang social donnant des privilèges, mais on peut les perdre. Au XIII siècle se pose le gros problème des clercs qui ont quitté toute fonction religieuse ou administrative pour des métiers très séculiers, commerçants ou artisans en particulier ; le concile de 1274 a tenté de résoudre en partie le problème par un biais, en excluant de la cléricature les "bigames ", c'est-à-dire, en général, ceux qui ont épousé une veuve; il semble que ce soit le cas de notre auteur, d'après ce qu'il dit ${ }^{26}$. Il faut cependant accueillir ces confidences avec une certaine méfiance : le thème du «clerc bigame destitué de ses privilèges ", assumé par un auteur pour ses amis (le Jeu de la Feuillée d'Adam de la Halle) ou pour son compte personnel («Matheolus », célèbre par l'adaptation française de Jean Le Fèvre), semble avoir été alors un topos littéraire (ou plutôt, dirions-nous aujourd'hui, médiatique) visant à attirer la sympathie des lecteurs laïcs vers des gens dont ils se sentent proches, et qui peuvent souffrir de la jalousie de leurs anciens camarades d'étude restés étroitement inféodés aux institutions cléricales.

19 À cette époque, il y a bien des « clercs » dans les métiers séculiers; en outre, il y a une inflation de « clercs du roi », des fonctionnaires qui ont des privilèges propres. Le «bon clerc» visé ici doit être, en principe, un clerc qui est resté dans des fonctions ecclésiastiques, ou plutôt juridiques (« en lois »), car les ecclésiastiques, en principe, sont devenus prêtres ou chanoines. Cependant, dans la première branche, quand Ysengrin consulte Renart sur le "parchemin » de la chèvre, il justifie les capacités de $l^{\prime}$ expert en lui disant ${ }^{27}$ :

Vous estes clerc, sans vanité ;

S'avez leu la divinité

Qui esclaircist les obscurtés

Et mes en paix toutes durtés

Et toute l'escripture expose.

20 La majorité des étudiants se spécialisaient dans le droit au XIV siècle, mais la science théologique («divinité») est ici posée (non sans quelque ironie) comme la base indiscutable de toute connaissance. La réponse de Renart, dans ce passage, dévie curieusement ce problème des connaissances vers l'« astronomie » (v. 2393), laquelle, en effet, occupe une place inattendue dans les développements de Renart le Contrefait sur les vices et les vertus ${ }^{28}$ - mais dans la bouche du milan Hubert, qui sera peut-être là le modèle du clerc enfermé dans les doctrines et les institutions de l'Église.

Dans notre passage, on pourrait donc penser que la maxime de Renart reproche à un clerc trop « clérical » une sorte de blocage de la communication, dans la mesure où sa situation tend à isoler un monde laïc d'un monde clérical. Ici, le blocage paraît 
particulièrement paradoxal : le baptême, rite religieux, est considéré comme une sorte de cérémonie intérieure à la culture laïque, qui lui impose sa langue, puisque le nom de baptême est à lire en français (fait présupposé comme normal, bien que liturgiquement étrange !). On pourrait donc imaginer que Renart rêve d'un super-clerc, qu'il n'est pas, et qu'Ysengrin n'est pas non plus, un clerc qui saurait lire le français comme le latin, et favoriser ainsi la communication.

Cependant, il faut tenir compte, à l'opposé, de la méfiance de la mentalité de l'époque pour les excès de la communication. Citons seulement la fable Le loup et le chien dans l'Ysopet II de Paris ${ }^{29}$ : le collier ne sert pas à empêcher le chien de mordre, mais au contraire à éviter qu'il sympathise avec des personnages extérieurs; ainsi que le texte de Guiot de Provins sur les Grandmontins, qu'il félicite de mettre les moines espagnols dans les abbayes de France et de Bourgogne, et inversement, pour que la différence de langue empêche la «connaissance» ou «l'acointance» avec les gens du pays ${ }^{30}$. Ici, le tort d'Ysengrin semble être d'avoir accepté le contrat avec une puissance laïque, représentée par la jument. En principe, «chacun de son côté »! Renart le suggère-t-il par ironie ? En fait, il y a plusieurs passages de Renart le Contrefait qui développent ou illustrent cet « isolationnisme », en particulier dans la cinquième branche de la seconde version, quand le moineau Drouin explique à Brun qu'il a eu tort de s'attaquer à Renart, car chacun doit s'abstenir de « jouer » avec des gens d'une autre condition ${ }^{31}$.

Dans la pratique institutionnelle de l'époque, des clercs peuvent être anoblis à condition de ne pas être chevaliers ${ }^{32}$. Un texte comme celui d'Henri de Crissey met à peu près sur le même plan les «langues " des laïcs de chaque peuple, et la langue unique des clercs, considérés comme une sorte de nation internationale ${ }^{33}$. Humbert de Romans parle de ceux qui oublient leur langue quand ils sont imprégnés dans une autre $^{34}$ : n'est-ce pas, pour Renart, le cas du clerc qui, plongé dans son latin, a oublié "suam linguam pro parte»? Reste à voir si c'est bien un mal dans la perspective d'Humbert : le clerc ne doit-il pas oublier sa culture laïque, et éviter les contacts avec des milieux éloignés de sa compétence? Il n'est pas impossible que, pour Renart, les clercs aient raison - ou croient avoir raison ! - de se tenir à l'écart du monde laïc, mais que les circonstances de l'époque les entraînent dans un engrenage où la technique de l'écriture est annexée par ce monde laïc, pour sa langue propre, si bien que le clerc ne peut plus lier cette technique à son enfermement dans la sécurité de sa langue savante : "S'Isangrin lire ne seüst / encore ceste prune il n'eüst ».

Le contexte de la maternité et du baptême

24 Pourtant, dans l'épisode de la chèvre, situé au début des deux versions, malgré le contexte narratif apparemment analogue, notre épicier gomme le problème de la langue savante et de la langue vulgaire, abordé dans l'épisode de la première version. Mais, si on y regarde de près, le cadre n'est pas le même.

Pour notre optique de linguistes, il n'y a pas un rapport étroit bien évident entre l'opposition langue savante/langue vulgaire et un contexte narratif associant la maternité, la filiation et le baptême. Et pourtant, ce n'est sans doute pas fortuitement que la sentence de Renart prend place dans un «rite familial». La problématique narrative présente ici une mère qui défend son enfant contre un prédateur, en écartant celui-ci grâce au rite qui est fait pour arracher l'enfant aux puissances démoniaques; mais ce rite, selon une évolution qui est secondaire pour les théologiens (pour eux, «baptiser » ne veut pas dire " donner un nom »!), s'est fixé, pour les laïcs, au niveau de l'inscription de l'enfant sous une étiquette individuelle chargée de force surnaturelle et 
prise dans le registre qui définit l'appartenance au clan. Même si le nom choisi paraît un signe arbitraire situé aux franges ou en dehors de l'éponymie, ou s'il évoque une connaissance encyclopédique diffuse, il se rattache à un idiome lié à la tradition familiale ou à la volonté d'expansion de la famille, plus qu'à une idéologie savante.

Une prise de conscience nouvelle des rapports complexes entre le problème des registres d'expression et celui des groupes d'appartenance collective (familiale et politique) semble donc avoir entraîné, à peu près à la même époque (XII $-\mathrm{XIII}{ }^{\mathrm{e}}$ siècle), le développement d'un système de repérage anthroponymique à deux niveaux (nom de baptême et nom de famille) et la définition de la langue vulgaire, en tant que telle, dans un registre plus nettement fotal, quand les formules du type «sermo paternus » sont brusquement remplacées par le type «sermo maternus " ${ }^{35}$. Le problème des niveaux de langue est ainsi lié, dans une pensée préconsciente, à celui des relations familiales ; dès le début $\mathrm{du} \mathrm{xII}^{\mathrm{e}}$ siècle, l'apparition de "sermo maternus » chez Guibert de Nogent n'avait pas seulement un lien évident avec l'alternative familiale des carrières dans le De vita $s u a^{36}$, mais elle était associée, peu logiquement pour nous, à la bigamie de Philippe I $^{\text {er }}$ (liée aux problèmes lignagiers) dans les Gesta Dei per Francos (où le pape Urbain II aurait pu être tenté d'évoquer dans sa langue « maternelle » française l'affaire mettant en cause la filiation royale dans son pays natal).

Renart, savamment "contrefait» par l'apothicaire de Troyes, ne pose donc pas le problème du français et du latin à propos de quelque rédaction d'un acte notarié, d'une délibération communale, ou d'un "recipe » à exécuter pour un de ses clients, mais à propos d'une maternité et d'un baptême. Et ce n'est pas un hasard si la seule histoire renardienne, inconnue pourtant du «Roman de Renart», qui est abondamment diffusée dans les contes populaires modernes, est celle de "Renart parrain", où le détenteur de la parole subversive invente à son profit personnel des dénominations baptismales ramenant l'arbitraire anthroponymique à un cynisme égocentrique qui détruit les bases spirituelles de la maternité et de la filiation ${ }^{37}$. Moins subversif, Renart "contrefait » laisse Ysengrin se faire simplement éliminer d'un lignage où il veut s'insérer indûment; et ce Renart-là s'enferme par jeu dans son latin de «légiste ». Le rite baptismal, celui dans lequel s'incarnent le plus profondément l'intégration de l'individu à l'Église et son intégration à un lignage, est par excellence le seuil où s'ouvre un choix entre l'intégrisme apparemment stérile d'une langue où la pesanteur juridique est encore plus forte que la charge sacrée, et une périlleuse ouverture sur un monde où la filiation physiologique est liée aux renouvellements de la vie, se traduisant dans la parole et même dans l'écriture par des accès de créativité difficiles à saisir pour le spécialiste des modèles fixes.

Peut-être faut-il remarquer, en marge, que ces modèles fixes sont masculins, et que le choc avec un monde biologique et laïc est ici une rencontre avec la féminité. La "langue maternelle " parle sous le pied de la jument, non dans la bouche du lion, et pourtant elle est paradoxalement la langue du roi $^{38}$ : la dualité des sexes et la dualité des langues sont dans un rapport sous tension.

Prenons le cas de Brunet Latin. De toute évidence, d'après le contexte, Dante le met en enfer comme pédophile, mais ce n'est pas sans raison que certains critiques ont pu prétendre que c'était parce qu'il avait écrit en français. Il y avait peut-être, dans l'attirance du pratiquant d'une langue vulgaire pour une autre langue vulgaire, quelque chose qui était parent de l'homosexualité, du refus de la hiérarchie conjugale. Dante, lui, est très net : il faut respecter le latin et respecter sa propre langue maternelle, mais 
il ne faut pas mélanger celle-ci avec d'autres langues vulgaires; il faut au contraire la promouvoir en vue d'une union conjugale avec le latin.

Que fait ici notre Renart? Il affirme la dualité sexuelle qui oppose le latin et le roman. Les deux langues sont pour lui séparées, et pas encore mariées. En proposant un baptême fondé sur la dénomination en langue "maternelle ", la jument propose une culture féminine, en face de laquelle Renart se dérobe, pour affecter de s'enfermer dans la culture mâle, la culture latine. À la même époque, la France affirme son désir d'une royauté en lignée masculine (on ne parle pas encore de "loi salique», mais l'idée est là). Bien sûr, sous Philippe le Bel, elle s'est détachée du pape, mais ce n'est pas pour s'enfermer dans une culture féminine; elle tient le roi en réserve comme garantie de masculinité. La connaissance "ès lois » de Renart paraît archaïquement fixée sur le latin de l'enseignement universitaire, mais, dans la pratique, elle est en train de se transposer sur la langue du roi à cette époque où de plus en plus d'actes juridiques ou politiques sont rédigés en français. La récupération de la culture livresque par le français autour de Charles $\mathrm{V}$ achèvera le travail. Mais la féminité de notre jument gardera en réserve une possibilité de domination sur la langue. "Quelle langue parlaient vos voix? - Meilleure que la vôtre !» Jeanne rejettera le Cauchon ou le loup qui prétendra la juger dans le registre de ces « machos " qui ne savent pas comment on apprend aux petits enfants à parler un «bon » français. Et ces machos sont des clercs : paradoxalement, le conte Aarne-Thompson $47 \mathrm{~B}$, qui représente toujours le triomphe de la sphère animale acquise à l'homme sur celle qui reste sauvage (ce n'est jamais un cerf qui donne le coup de sabot, ni un chien qui le reçoit!) insinue ici que cette sauvagerie, dans l'humanité, est du côté du clergé, et que la vraie « culture » est donc du côté des laïcs - représentés par un personnage féminin...

Mais qu'est-ce que ça veut dire?

31 Nous venons de frôler, derrière le texte, des arrière-plans idéologiques ou psychologiques préconscients (parlons, si l'on veut, d'un " plan translocutionnaire »!) et nous ne serons pas très sûrs de bien redescendre au niveau "perlocutionnaire " traditionnel, pour chercher les intentions prêtées à Renart par le narrateur et celles qu'il assume lui-même. Mais faut-il s'y risquer? Dans Renart le Contrefait, le discours du héros est un discours ouvert et souple - comme chez la plupart des très grands écrivains... Notre auteur a-t-il des intentions précises? On pourrait penser, par exemple, qu'il a un compte à régler avec des clercs latinisants de sa connaissance (qui tentent maladroitement d'écrire en français, alors que lui, pourtant déchu de leurs privilèges, sait le faire habilement...) ; les règlements de compte personnels sont à la mode dans la littérature de l'époque, et pas toujours aussi explicites que chez Dante. Mais on pourrait penser aussi, quand on a lu tout Renart le Contrefait, que l'auteur a plaisir à dire tout ce qui lui passe par la tête ou qu'il trouve dans ses manuscrits, sans souci de cohérence ni d'inscription sur le compte d'un porte-parole positif ou négatif.

Si l'on veut chercher une sorte de cohérence dans ses idées, on rencontre la théorie de J. Scheidegger, selon lequel l'ouvrage entier est une entreprise de mise en dérision des connaissances $^{39}$. Cette vue est peut-être assez juste à la limite, mais il ne faut pas la pousser en imaginant un parti pris très systématique et volontaire d'un épicier blasé sur la qualité profonde de ses drogues; s'il remet en question la science, c'est dans la mesure où les clercs veulent s'en faire un privilège, mais il ne force pas la critique sociale. Je suggérerais plutôt qu'il s'inspire du Roman de Renart pour mettre tout sur le plan du jeu. On peut donc jouer au clerc, jouer au laïc, jouer au latin, jouer au français : 
en jouant au clerc, Ysengrin a perdu, et Renart s'est situé hors-jeu. Le décepteur n'est-il pas, au fond, celui qui a conscience de l'inadéquation de sa conduite, et qui la poursuit quand même à titre de jeu ${ }^{40}$ ? Bien sûr, une vision ludique de la diversité des langages n'est pas une règle générale à l'époque, mais c'est peut-être une possibilité limite. Nous savons combien la langue du XIV siècle est souvent bien ambiguë et un peu confuse dans sa phonétique, son vocabulaire, et surtout sa syntaxe; nous pourrions dire qu'elle est même ambiguë quand elle parle d'elle-même.

\section{NOTES}

1. On peut penser au « marginal man » de certains sociologues américains; pour un modèle animal de cette situation ambiguë, voir J. Batany, « The marginal beast : la chauve-souris des fables et l'ambiguïé d'un statut », Reinardus, 14, 2001, p. 3-22. 2. Le Roman de Renart de Contrefait, G. Raynaud et H. Lemaître éd., Paris, 1914 (2 vol.). Cette édition est celle de la deuxième version, mais elle donne en notes de larges passages de la première. On pourra prendre un premier contact avec l'œuvre à travers le chapitre que lui consacre Ch.-V. Langlois, La vie en France au Moyen Âge d'après des moralistes du temps, $2^{e}$ éd., Paris, 1926, p. 291-320.

3. Sur une de ces fables, voir J. Batany, « Le rat de ville et le rat des champs : traditions littéraires et conjonctures sociales ", Bien dire et bien aprandre, 5, 1987, p. 27-46. Cet apologue précède le nôtre et pourrait aider à le comprendre, mais en tenant compte des entrecroisements sémiotiques entre les deux récits au lieu de chercher des équivalences simplistes (Le Roman de Renart de Contrefait, op. cit., t. II, p. 239-241). 4. L'Ésope de Julien Macho, P. Ruelle éd., dans Recueil général des Isopets, t. III, Paris, 1982, p. 112-113 («Du lyon et du cheval»), p. 147-149 (« Du mullet, du regnard et du loup ») et p. 167-168, comme épisode d'un conte à rebondissements (voir à ce sujet mon article "Chaîne de fables et revue d'estats : le loup qui fit un gros pet ", Reinardus, 4, 1991, p. 3-18).

5. Il s'agit du conte-type $47 \mathrm{~B}$ du recueil d'A. AARNE et S. THOMPSON, The Types of the Folktale, Helsinki, 1961.

6. Le Roman de Renart de Contrefait, op. cit., t. II, p. 242, v. 24-27 de la première colonne ; dans les extraits de la première version, l'édition ne numérote pas les vers.

7. Ibid., p. 243 (1 $1^{\text {re }}$ colonne, v. 5-12).

8. Ibid., v. 16-20.

9. Ibid., t. I, première branche, v. 995-3185, p. 11-34. L'histoire de Renart, Ysengrin et la chèvre se retrouve en deux versions apparentées dans les Récits d'un ménestrel de Reims et dans Rainaldo e Lesengrino, mais sans le motif du parchemin à déchiffrer. Voir A. Lomazzi, Rainaldo et Lesengrino, Florence, 1972, p. 32-37 et 170-182.

10. Voir J. Batany, «Écrit/Oral » dans Dictionnaire raisonné de l'Occident médiéval, J. Le Goff et J.-Cl. Schmitt dir., Paris, 1999, p. 309-323 ; et, bien entendu, P. Zumthor, La Lettre et la voix. De la « littérature » médiévale, Paris, 1987.

11. S. Lusignan, Parler vulgairement. Les intellectuels et la langue française aux XIII et XIV siècles, Paris-Montréal, 1987. 
12. Dante, De vulgari eloquentia, P. V. Mengaldo éd., Padoue, 1968.

13. Ibid., I, X, 2, p. 16-17.

14. Voir, par exemple, Y. Cazal, Voix du peuple/Verbum Dei. Contribution à une étude du bilinguisme latin/langue vulgaire au Moyen Âge, Genève, 1998.

15. Voir C. Marchello-Nizia, Histoire de la langue française aux $X^{2} V^{e}$ et $X V^{e}$ siècles, Paris, 1979, et les travaux auxquels elle renvoie (M. Delbouille, Th. Gossen, etc.).

16. Voir J. Batany, «L'amère maternité du français médiéval », Langue française, 54, mai 1982, p. 29-39, repris dans Approches langagières de la société médiévale, Caen, 1989, p. 95-105.

17. Sur ce point, voir J.-P. Makouta-Mboukou, Le Français en Afrique Noire, Paris, 1973.

18. S. Lusignan, Parler vulgairement, op. cit., en particulier p. 35-47.

19. Roger Bacon, Opus tertium, XXV, cité par S. Lusignan, Parler vulgairement, op. cit., p. 73.

20. Voir Raymond Lulle, Doctrine d'enfant, A. Llinares éd., Paris, 1969, p. 27 (phrase citée d'après le texte catalan, mais supprimée par le traducteur français du Moyen Âge : elle se référait à un texte qu'il n'éditait pas).

21. Voir S. Lusignan, Parler vulgairement, op. cit., p. 129-172.

22. Le Roman de Renart de Contrefait, seconde version, $2^{\mathrm{e}}$ branche, op. cit., t. I, p. 202.

23. Francine Mora, spécialiste de la descendance médiévale de l'Enéide, m'a aimablement fait part d'un sondage négatif sur ce point. Pour les débats sur l'origine du latin, voir A. Borst, Der Turmbau von Babel, Stuttgart, 1957-1963 (6 vol.).

24. Jehan Le Teinturier D'Arras, Le Mariage des sept arts, A. Langfors éd., Paris, 1923, p. 102-103.

25. Voir S. Lusignan, Parler vulgairement, op. cit., p. 62-66.

26. Le Roman de Renart de Contrefait, op. cit., t. I p. 34, v. 3191-3196 (texte propre à la seconde version).

27. Ibid., $2^{\mathrm{e}}$ version, $1^{\text {re }}$ branche, t. I, v. 2359-2363.

28. Ibid., t. II, v. 34309-36199.

29. Recueil général des Isopets, t. I, J. Bastin éd., p. 171-173, fable XXVI ; la source semble être la fable latine d'Alexandre Neckam, où le chien, au contraire, expliquait son collier par sa méchanceté envers les étrangers (voir J. Batany, « Une liberté ambiguë : le loup et le chien ", Reinardus, 12, 1999, p. 3-17).

30. Voir Les œuvres de Guiot de Provins, J. Orr éd., Manchester, 1915, p. 57, v. 1521-1532. 31. "Noble, clerc a ruddes villains/Envis poeut estre bon compains ;/Le clerc latin parler voulroit,/Et le vilain mal le soulroit,/Car le vilain ne s'estudie/Fors qu'en charue et en boulye » (Le Roman de Renart de Contrefait, op. cit., t. III, p. 83, v. 30481-30486).

Drouin raconte à l'appui une anecdote où l'âne Bernard a eu le tort d'accompagner Renart ; celui-ci est acquitté pour avoir suivi sa nature en pillant le poulailler, alors que l'âne est puni pour avoir brouté du gazon au lieu de ses chardons habituels : " Pour ce est fol qui voeult compagnie/S'il ne scet tresbien de leur vie/Et de quel condicion est » (p. 84, v. 30539-30541).

32. Voir R. Cazelles, La Société politique et la royauté sous Philippe de Valois, Paris, 1958, p. 320.

33. Cité par S. Lusignan, Parler vulgairement, op. cit., p. 41 : langues maternelles et langue latine sont toutes artificielles (« voces impositae ad placitum »). Il faudrait peut-être réexaminer les idées de ce texte, qui semble concevoir une société pluriculturelle plus que diglossique. 
34. Humbert de Romans, De eruditione, VII, 39, cité d'après S. Lusignan, Parler vulgairement, op. cit., p. 57. L'interprétation de ce dernier, pour qui ce texte présenterait la langue française comme «meilleure que les autres ", est peut-être à revoir.

35. Voir J. Batany, «L'amère maternité du français médiéval », loc. cit., et « Langages et identités culturelles dans la France médiévale ", dans Langue française et identités culturelles (VII ${ }^{\mathrm{e}}$ Biennale de la langue française), Dakar, 1985, p. 154-167, repris dans Approches langagières, op. cit., p. 77-94.

36. Voir J. Batany, «L'autobiographie de Guibert de Nogent : identité sociale et identité langagière ", dans Autobiographie et références autobiographiques au Moyen Âge, D. Buschinger éd., Greifswald, 1995, p. 1-10. Guibert se montre fier d'avoir su s'exprimer devant le Pape (à propos de la Commune de Laon) non pas dans sa langue vulgaire, le " sermo maternus » auquel sa mère voulait le réduire quand elle prétendait l'arracher, dans son enfance, à un précepteur brutal, mais en latin, montrant ainsi sa pleine intégration à la carrière cléricale à laquelle l'avait voué son père défunt.

37. Rappelons l'histoire de « Renart-parrain » (type 15 du recueil Aarne-Thompson) : Le renard et le loup ont mis de côté une réserve de nourriture, et s'en vont travailler ensemble, mais le renard s'absente trois fois en prétendant qu'on l'appelle pour être parrain à un baptême, et il en profite pour manger en cachette une partie de la réserve ; le loup lui demande à chaque fois le nom qu'il a donné à son filleul, et le renard répond : «Bien-Entamé », « Moitié-Vide », « Bien-Reléché ». Ce conte est souvent l'introduction d'une chaîne d'aventures où le renard dupe le loup. Voir P. Delarue - M.L. Ténèze, Le Conte populaire français, Paris, 1976, t. III, p. 68-69, 106-109, et 295-303 (92 versions !). Sur le rapport entre les « échecs du loup » et le baptême, voir aussi mon article, "Chaîne de fables et revue d'estats... ", loc. cit.

38. Voir J. Batany, «L'amère maternité du français médiéval », loc. cit., et « Langages et identités culturelles dans la France médiévale », loc. cit.

39. J. Scheidegger, Le Roman de Renart ou le texte de la dérision, Genève, 1989, p. 338-359 (« Le savoir contrefait»).

40. Voir entre autres J. Batany, Scène et coulisses du Roman de Renart, Paris, 1989, p. 23-45 («Un faux type universel : le décepteur »).

\section{RÉSUMÉS}

La première version de Renart le Contrefait (vers 1325) rapporte un conte où le loup ne peut déchiffrer sous le pied de la jument le nom du poulain qu'elle veut lui faire baptiser, et où Renart se récuse pour l'aider : il n'a fait que des études juridiques, dit-il, et un «bon clerc » ne sait pas lire ni écrire en « roman ». La boutade n'envisage pas la langue vulgaire sous sa forme orale, mais sous un jour écrit, où elle peut paraître paradoxalement plus difficile que le latin, pourtant vanté ailleurs dans l'ouvrage. Mais le clerc a-t-il tort de restreindre la communication en s'enfermant dans le latin ? Le contexte de la maternité et du baptême éclaire ou complique ici le problème, et évoque le choc entre « clergie » et féminité. Mais ce texte est un jeu, comme tout le roman, et sa fantaisie peu cohérente ne cherche guère une dérision systématique de la « clergie ». 
Clerics and Romance Languages : a 14th Century Jest from Reinart the Fox. The first version of Renart le Contrefait (around 1325) tells the story of a wolf that cannot decipher the name of the foal that is written under the hoof of the mare that wants to have it baptised. Renart declines to help on the grounds that he had only studied law and that a "good cleric» is not able to read or write in "roman ». The jest does not envisage the common language as speech but only in its written form where, paradoxically, it could appear to be more difficult than Latin although the latter is nevertheless praised elsewhere in the work. But is the cleric wrong to limit communication in enclosing himself in Latin? The context of maternity and baptism both throws light on and complicates the problem, and evokes the shock of the encounter of clerkdom and femininity. This text is, however, a game, as is the whole book, and its somewhat incoherent fantasy does not really aim at a systematic derision of the clerkdom.

INDEX

Mots-clés : ancien français, clergie, fable animale, langue maternelle

Keywords : Old French, Renart le Contrefait, animal fable, clerkdom, native language

\section{AUTEUR}

\section{JEAN BATANY}

Université de Caen, 332, rue Lecourbe, F-75015 Paris. 